Vorwort und Einleitung zum Gesamtwerk sind in beiden Bänden übereinstimmend abgedruckt.

Das Schwergewicht des Werkes liegt auf der politischen Geschichte, die allerdings im Islam noch weit enger mit der Religion verbunden ist, als es im Christentum der Fall war. Von besonderem Interesse dürften dabei jeweils die Schlußteile beider Bände sein. Der Teil 4 (Die Islamischen Zentralgebiete in der Gegenwart) umfaßt folgende 7 Kapitel: Die moderne Türkei, die arabischen Länder, das moderne Persien, Islam in der SU, Kommunismus in den islamischen Zentralgebieten, der politische Einfluß des Westens, wirtschaftlicher und sozialer Wandel. Der Teil 8 (Islamische Gesellschaft und Zivilisation) gliedert sich in folgende $13 \mathrm{Ab}$ schnitte: geographischer Umfang, Quelle islamischer Zivilisation, Wirtschaft Gesellschaft - Institutionen, Recht und Justiz, Religion und Kultur, Mystik, Wiederbelebung und Reform im Islam, Literatur (arabisch, persisch, türkisch, Urdu), Kunst und Architektur, Wissenschaft, Philosophie, Kriegführung, Übertragung von Gelehrtheit und literarischer Einfluß auf den Westen.

Hellmuth Hecker

\section{H. Dodp}

Politics and Government in Turkey Manchester University Press, 1969, XVI + 335 S., 65 s.

Die Schilderung der Innenpolitik während der Jahre 1961-1965, das heißt während der vierjährigen Legislaturperiode des ersten Parlaments nach dem Inkrafttreten der neuen Verfassung vom 9.7. 1961, bildet die Grundlage für eine ausführliche Darstellung der politischen Organisation und der Verwaltung der Türkei ,in action“. Wie der Verfasser betont, ist er weniger an den soziologischen und juristischen Strukturen als an den Institutionen und Persönlichkeiten interessiert, welche in der Politik bedeutsam sind und in den Zeitungen und Zeitschriftenartikeln sowie in Abhandlungen und Büchern $t \ddot{u} r-$ $\mathrm{k}$ is che $\mathrm{r}$ Autoren ihre Widerspiege- lung gefunden haben. Dementsprechend beschränkt sich das Verzeichnis der Primörquellen (Publikationen der politischen Parteien sowie der staatlichen Organe, Behörden und Anstalten, Interviews und Fragebogenaktionen des Verfassers) und der Sekundärquellen (ausschließlich türkische und englische Abhandlungen und Bücher) auf die Zeitspanne zwischen 1959 und 1965, obwohl das Werk erst im November 1969 erschienen ist. Das kurze Nachwort ändert nichts an dieser zeitlichen und sachlichen Beschränkung. Nichtsdestoweniger ist es dem Verfasser gelungen, die politische Organisation, d. h. die Verfassung, die politischen Parteien, die "Pressure Groups" (Armee, Studentenschaft, Gewerkschaften), den Ablauf der Wahlen, die Arbeit des Parlaments, des Ministerrats und des Präsidenten der Republik einerseits, den Verwaltungsaufbau, insbesondere das Zusammenspiel von Zentral-, Provinzial- und Lokalverwaltung, das Amterwesen und das Beamtentum andererseits in ihrer politischen Wirklichkeit, in ihrer Interaktion und Interdependenz anschaulich zu schildern, ohne den Ehrgeiz zu haben, hiermit eine Geschichte der türkischen Innenpolitik während der fraglichen Zeitspanne verfaßt zu haben. Wer das einschlägige, auf türkischen Quellen beruhende, deutschsprachige Schrifttum der letzten zwei Jahrzehnte kennt, wird in dem Buch von Dodd kaum etwas Neues finden, abgesehen vielleicht von Einzelheiten über die Richtungskämpfe innerhalb der politischen Parteien. Im Spiegel der augenblicklichen Ereignisse (d. h. im ersten Halbjahr 1971) bilden folgende zwei Sätze einen vollgültigen Beweis dafür, wie tief der Verfasser in die politische Wirklichkeit der Türkei eingedrungen ist: "But if the argument between left and right centre develops into a fight which breaks the bounds of the democratic system, the military might well intervene for sake of national solidarity and to clear up the mess.” (S. 319). „In a liberal democratic system in an underdeveloped society like that of Turkey the agreed means of changing governors must be shown 
to be real, which means that it must happen. Paradoxically, too much stability can be a dangerous thing."

Ernst E. Hirsch

\section{Al Tibawi}

A Modern History of Syria including

Lebanon and Palestine

London, Macmillan 1969, 441 S.

Rizkallah Hilan

Culture et dévelopment en Syrie et dans les pays retardés

Paris, éditions anthropos 1969. XV, $388 \mathrm{~S}$.

Zeitlich umfaßt das erste Werk die Spanne vom Anfang des 19. Jahrhunderts bis zur Erlangung der Selbständigkeit als Folge des Zweiten Weltkrieges. Diese Spanne ist unterteilt in die Phase des direkten und die des indirekten westlichen Kolonialismus. Die erste von ihnen reicht bis zum Zerfall des Türkischen Reiches: Für diese Zeit sieht der Verfasser trotz formeller türkischer Herrschaft Syrien in Wirklichkeit in der Hand der Engländer und Franzosen, angefangen von der Mission über die kulturelle Vorherrschaft bis zur wirtschaftlichen Ausbeutung. Die direkte Kolonialherrschaft beginnt 1919 mit der Maßgabe, daß zu Franzosen und Engländern (das Sykes-Picot-Abkommen wird S. $240 \mathrm{ff}$. behandelt) kraft der Balfour-Deklaration die Israelis hinzukommen. Der Verfasser sieht hierin einen Treubruch der beiden Völker gegen die arabische Welt: Frankreich und England hätten unter dem Versprechen von Selbständigkeit die Araber veranlaßt, sich gegen die Türken zu erheben, ihnen aber später die Selbständigkeit vorenthalten und zudem Syrien geteilt sowie ihm Palästina entfremdet. Dieser Vorwurf, der voll Bitterkeit immer wieder erneuert wird, hat sich durch die Erlangung der Selbständigkeit nicht etwa erledigt. Er dauert fort insofern, als Syrien immer noch geteilt und ihm Palästina immer noch genommen ist. Die geschichtliche Darstellung ist genau und gründlich. Nicht zuletzt leistet sie einen Beitrag $\mathrm{zu}$ einer Frühgeschichte der Modernisierung, gewissermaßen ih- rer ersten Welle: es handelt sich um den Anstoß, den Napoléons Expedition nach Ägypten zunächst für dieses selbst, dann aber für ganz Syrien bedeutet hat.

Hilan hat sein Buch der Gegenwart Syriens gewidmet, der allerdings eine Vorgeschichte von nicht weniger als 100 Seiten vorausgeschickt ist, die mit den ersten Kulturen dieses Landes einsetzt. Was die Gegenwart angeht, so sind hier politische Verfassung und politische Kräfte ausgeschaltet (331): Technik, Wirtschaft und Kultur sind die Themen, auf deren Behandlung der Verfasser sich beschränkt. Aber auch in diesen Bereichen beschäftigt er sich nicht mit Institutionen, Verfassungen und Normen, er begnügt sich vielmehr mit der Schilderung von Zuständen und Vorgängen. Von dem ersten erfährt man allenfalls, daß die neue (neu $=$ seit 1955) syrische Wirtschaftspolitik „s' inspire du modèle socialiste" (235); und selbst von den beiden Fünfjahresplänen (1960 ff.; 1965 ff.) ist nur sporadisch die Rede (vor allem 188 f.; 191; 236). In diesem auf die Faktizität beschränkten Rahmen sieht der Verfasser das Haupthindernis der Modernisierung in einem Mangel an technischem Sinn und an ebensolchen Fachkräften. Hierauf führt er in Verbindung mit der Unmodernität der Wirtschaftsgesinnung der Führenden Schichten (ihre Privatinitiative wird 170 als "essoufflé" bezeichnet) nicht nur das Untermaß, sondern vor allem auch die Art der Investitionen zurück: Statt fehlende, insbesondere Grundstoff- und Schwerindustrien aufzubauen, wird die bestehende Textilindustrie immer weiter vergröBert, obwohl der Weltmarkt gerade an dieser Stelle übersetzt ist. Diese Unzulänglichkeiten mindern sich, seitdem der Staat vor allem mittels der erwähnten zwei Fünfjahrespläne die Wirtschaft an die Hand genommen hat (236). Bemerkenswert ist, daß trotz alledem die Neigung $\mathrm{zu}$ naturwissenschaftlichen und technischen Studien gering bleibt: Nach wie vor überwiegen an den Universitäten die Geisteswissenschaftler und un- 